\title{
Curcumin plays a protective role against septic acute kidney injury by regulating the TLR9 signaling pathway
}

\author{
Huiqing $\mathrm{Li}^{1}$, Hui Sun ${ }^{2}$, Yaru Xu' ${ }^{2}$, Guihua Xing ${ }^{3}$, Xuehui Wang ${ }^{4}$ \\ ${ }^{1}$ Department of Nephrology, The First Affiliated Hospital of Qiqihar Medical University, Qiqihar, China; ${ }^{2}$ Department of Clinical Laboratory, The \\ First Affiliated Hospital of Qiqihar Medical University, Qiqihar, China; ${ }^{3}$ Ultramicropathology Experimental Center, Qiqihar Medical University, \\ Qiqihar, China; ${ }^{4}$ Department of Nephrology, General Hospital of the General Administration of Agriculture and Reclamation of Heilongjiang, \\ Harbin, China \\ Contributions: (I) Conception and design: H Li, Y Xu; (II) Administrative support: G Xing; (III) Provision of study materials or patients: H Li, X \\ Wang, H Sun; (IV) Collection and assembly of data: G Xing, H Li; (V) Data analysis and interpretation: X Wang, H Sun; (VI) Manuscript writing: \\ All authors; (VII) Final approval of manuscript: All authors. \\ Correspondence to: Xuehui Wang. NO. 235, Hashuang Road, Nangang District, Harbin 150088, China. Email: hebykdx1@163.com.
}

Background: This study aims to evaluate the inhibitory effect of curcumin (Cur) on the progression of septic acute kidney injury (SAKI), in order to improve the survival rate in this patient population.

Methods: Acute kidney injury (AKI) was induced by cecal ligation perforation (CLP) in Sprague-Dawley (SD) rats. Using this AKI animal model, the survival rate of the rats was evaluated at different time points after Cur treatment to explore whether Cur can improve survival in an animal model of AKI. The expression levels of inflammatory factors (NF- $\mathrm{B}, \mathrm{TNF}-\alpha$, and IL-10), organ injury markers [urea nitrogen (UN), creatinine $(\mathrm{Cr})$, alanine aminotransferase (ALT), aspartate aminotransferase (AST), amylase, creatine kinase (CK), and lactate dehydrogenase ( $\mathrm{LDH})$ ], and disease progression markers [neutrophil gelatinase-associated lipocalin (NGAL), kidney injury molecule-1 (KIM-1), and cystatin-C (CysC)] were determined using an enzyme-linked immunosorbent assay (ELISA).

Results: The serum levels of UN, Cr, NF- $\kappa$ B, ALT, AST, amylase, CK, LDH, inflammatory factors TNF- $\alpha$ and IL-10, and markers of early diagnosis of SAKI (NGAL, CysC, KIM-1) were significantly lower in the curcumin group than those in the placebo group $(\mathrm{P}<0.05)$. In addition, serum levels of TLR9 and its downstream molecules MyD88, IRF5, and IRF7 in the curcumin group were significantly lower than those in the placebo group $(\mathrm{P}<0.05)$. The application of TLR9-specific inhibitors to experimental rats led to similar results as those obtained in the curcumin group, whose detection indexes were significantly lower than those in the placebo treatment group $(\mathrm{P}<0.05)$.

Conclusions: Given the excellent performance of Cur in anti-tumor, anti-oxidation, anti-inflammatory, and other clinical trials, it is very likely to be further developed as a potential drug for the clinical treatment of AKI.

Keywords: Septic acute kidney injury (SAKI); survival rate; curcumin; TLR9 signaling pathway

Submitted Apr 02, 2021. Accepted for publication May 20, 2021.

doi: $10.21037 /$ tau-21-385

View this article at: http://dx.doi.org/10.21037/tau-21-385

\section{Introduction}

Acute kidney injury (AKI) is one of the most common and serious complications of sepsis patients in the intensive care unit (ICU). Patients often develop multiple organ failure, resulting in a mortality rate of $60-80 \%$ (1).
Toll-like receptors (TLRs) can recognize the nucleic acids of bacteria and viruses and trigger innate immune responses (2). MyD88 protein, an essential downstream adapter molecule in the mitochondrial activated protease and $\mathrm{NF}-\kappa \mathrm{B}$ signaling pathway, can activate most of 
the TLR signals (3). Studies have shown that MyD88deficient rats have good resistance to the development of sepsis, but TLR2- and TLR4-deficient rats do not show resistance (4). TLR9 knockdown significantly inhibited renal cell apoptosis, alleviated the severity of sepsis induced acute kidney injury, and improved the survival rate of rat model (5).

Curcumin (Cur) is a dietary polyphenol extracted from the Zingiberaceae family of plants. Cur has been shown to have good clinical value in cancer (6), osteoarthritis (7), anxiety and depression (8), dyslipidemia (9), metabolic syndrome (10), respiratory diseases (11), nonalcoholic fatty liver (12), type 2 diabetes (13), and ulcerative colitis (14).

Although Cur has been studied in a variety of human disease models, its application in septic acute kidney injury (SAKI) has not been thoroughly explored. In this study, we used a Sprague-Dawley (SD) rat model of SAKI to explore the inhibitory effect of Cur on this disease from different angles. Our results preliminarily proved that Cur exerts its inhibitory effect on SAKI by inhibiting the TLR9 signaling pathway, which provides a theoretical basis for the clinical application of Cur in SAKI.

We present the following article in accordance with the ARRIVE reporting checklist (available at http://dx.doi. org/10.21037/tau-21-385).

\section{Methods}

\section{Experimental materials and instruments}

Specific pathogen-free (SPF) SD rats (32-40 weeks old) were purchased from Shanghai Xipuer-Bikai Laboratory Animal Co., Ltd. Cur was purchased from SIGMA Biological Company, USA (batch No. 08511). The Cur powder was dissolved in normal saline to prepare a $400 \mathrm{mg} / \mathrm{mL}$ solution, which was then filtered by a 0.22 um sterile filter membrane and stored at $-20{ }^{\circ} \mathrm{C}$ for later use. Purchased from InvivoGene (France), the TLR9-inhibitor and inhibitor-control drugs (batch Nos. ODN 2088 and ODN 2088 control) were prepared according to the method for Cur and stored after filtration. Isoflurane was purchased from Baxter Caribe company, USA (batch No. N094M209). The NF-kB-specific ELISA kit was purchased from Abcam Co., USA (batch No. ab176663). ELISA kits specific for tumor necrosis factor (TNF)- $\alpha$, interleukin (IL)10, neutrophil gelatinase-associated lipocalin (NGAL), cystatin-C (CysC), and kidney injury molecule-1 (KIM-1) were all purchased from Wuhan Boster Biological
Technology Co. Ltd. (batch Nos. EK0527, EK0417, EK0854, EK0880, and EK0679, respectively). ELISA kits specific for TLR9, MyD88, and IRF5 were purchased from Wuhan FineTest Biological Company (batch Nos. EM1412, EM0669, and EM0667, respectively). The IRF7 ELISA kit was purchased from LifeSpan Biological Company, USA (batch No. LS-F53197). Other commonly used biochemical reagents were purchased from Shanghai Beyotime Biotech Co. Ltd.

The 5424R high-speed refrigerated microcentrifuge was purchased from Thermo Fisher Scientific, USA (Model 917), the automatic biochemical analyzer from Hitachi, Japan, and the Model 680 full-wavelength microplate reader from Bio-Rad Company, USA.

\section{Survival rate assessment experiment}

Experiments were performed in compliance with Qiqihar Medical University guidelines for the care and use of animals. The study was approved by the Qiqihar Medical University Institutional Review Board (No. QMUAECC-2018-32), Heilongjiang, China. Twenty-four SPF $\mathrm{SD}$ rats in the same batch were randomized into groups $\mathrm{A}$, $\mathrm{B}$, and $\mathrm{C}$, with eight rats in each group. Group A (curcumin group) underwent cecal ligation perforation (CLP). The rats were given Cur orally $(40 \mathrm{mg} / \mathrm{kg}) 6$ hours after the operation. Group B (placebo group) was given the same dose of normal saline 6 hours after CLP. Group C (sham group) underwent a sham operation. The experimental animals were observed 24, 48, 72, and 96 hours after treatment for survival status, and were euthanized when they became seriously ill.

The CLP procedure was as follows: After isoflurane anesthesia and laparotomy, 4-0 ligation silk thread was fastened $15 \mathrm{~mm}$ away from the cecal tip of the SD rats. Then, the cecum was punctured twice with a 21-gauge needle, and a small amount of fecal excretion was gently extruded and inserted into the center of the abdominal cavity. At the end of the operation, $20 \mathrm{~mL} / \mathrm{kg}$ of normal saline was injected subcutaneously. For the sham group, CLP was not performed, but the other procedures remained unchanged. After surgery, the abdominal incision was sutured with 6-0 nylon thread (15).

\section{Animal model index detection}

Survival rate evaluation experiments were conducted, then blood samples $(1.0 \mathrm{~mL})$ and urine samples $(0.5 \mathrm{~mL})$ were 
collected from the tail veins of the animals after 24 hours. After the blood samples were left at room temperature for 20 minutes, the blood and urine samples were centrifuged at 2,000 $\mathrm{rpm} / \mathrm{min}$ for 20 minutes to collect the serum and urine supernatants, which were then packaged and frozen at $-20{ }^{\circ} \mathrm{C}$ for the following experiments (16).

\section{Evaluation of renal function injury}

The levels of urea nitrogen (UN), creatinine (Cr), and the expression of inflammatory protein $\mathrm{NF}-\kappa \mathrm{B}$ in the collected animal serum samples were detected to evaluate the degree of kidney injury in the different treatment groups.

* UN detection: a Hitachi 917 automatic biochemical analyzer was used to detect the amount of UN in the collected serum samples.

* Cr detection: the detection of $\mathrm{Cr}$ in the serum was determined by the picric acid-based colorimetric kinetic assay using the Astra 8 autoanalyzer.

* NF-кB protein detection: the protein expression level of $\mathrm{NF}-\kappa \mathrm{B}$ in the serum was detected by an ELISA kit from Bio-Rad, USA (450 ELISA).

\section{Evaluation of functional impairment of other organs}

The changes in alanine aminotransferase (ALT), aspartate aminotransferase (AST), amylase, creatine kinase (CK), and lactate dehydrogenase $(\mathrm{LDH})$ in the serum samples were detected to evaluate the functional impairment of other organs in the different treatment groups. A Hitachi 917 automatic biochemical analyzer was utilized to measure the indexes in the collected serum samples.

\section{The degree of immune reaction in the different treatment groups}

The changes in TNF- $\alpha$ and IL-10 in the serum samples were detected to evaluate the changes in inflammatory factors. The detection was carried out by TNF- $\alpha$ - and IL10-specific ELISA kits.

\section{Change trend of AKI markers}

The molecular expression levels of NGAL, KIM-1, CysC, and other markers in the urine samples were detected to provide a reference for the clinical development of AKI. NGAL-, Kim-1- and CysC-specific ELISA kits were used for the detection.

\section{Detection of TLR9 expression}

By detecting TLR9 expression in the serum samples, the activation degree of the TLR9 signaling pathway in the development of SAKI was preliminarily determined. A TLR9-specific ELISA kit was used for the detection.

\section{Mechanism exploration}

Six hours after CLP, the SD rats were randomly assigned into three groups, which were given equal doses of Cur (40 mg/kg), TLR9 inhibitor (ODN 2088), and the control drug (ODN 2088 Control), respectively. After 24 hours, serum UN, Cr, ALT, AST, amylase, and CK levels were determined according to the above experimental protocol.

Meanwhile, MyD88, IRF5, and IRF7 levels in the collected serum samples were detected by MyD88-, IRF5and IRF7-specific ELISA kits.

All tests were conducted in strict accordance with the manufacturers' instructions.

\section{Statistic analysis}

GraphPad Prism 7.0 software was used for the data processing, and all statistical data were expressed as the mean \pm standard error (mean \pm SE). Differences between the experimental groups were analyzed by multiple comparisons and correction analysis of variance or $t$-tests for statistical significance. The level of significance was taken as $\mathrm{P}<0.05$.

\section{Results}

\section{Cur significantly reduces $S A K I$-induced mortality}

A significantly improved survival rate was observed in rats who were treated with Cur 6 hours after CLP. Compared with $80 \%$ of the rats in the curcumin group, only $40 \%$ of the rats in the placebo group survived 96 hours after the operation (Figure 1).

\section{Cur is beneficial in alleviating SAKI-induced kidney damage}

To explore the protective effect of Cur on the kidneys, we tested the related factors. The levels of UN, Cr, and NF$\kappa \mathrm{B}$ in the serum of the SD rats in the curcumin and sham groups were found to be significantly lower than those in the placebo group $(\mathrm{P}<0.05)$, indicating that Cur treatment 


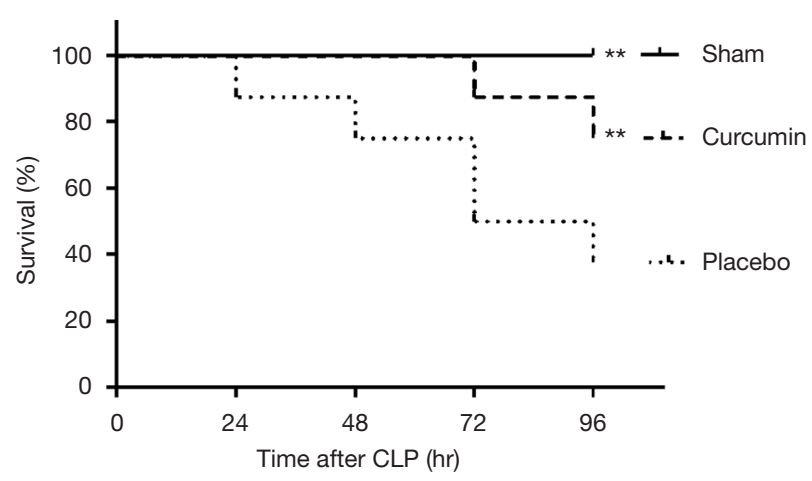

Figure 1 Cur significantly reduces SAKI-induced mortality in rats after CLP. Survival curves of SD rats undergoing CLP were given different treatments 6 hours after surgery: The solid line represents the sham group $(n=8)$, the dotted line represents the curcumin group ( $40 \mathrm{mg} / \mathrm{kg}$ ), and the chain-dotted line represents the placebo group (equal volume of normal saline). ${ }^{* *}$ indicates $\mathrm{P}<0.01$, with the placebo group as the control. SAKI, septic acute kidney injury; SD, Sprague-Dawley; CLP, cecal ligation perforation.

6 hours after surgery can effectively alleviate kidney injury caused by sepsis (Figure 2).

\section{Cur has a good protective effect on many organs}

The levels of ALT, AST, amylase, CK, and LDH in the serum samples of the curcumin and sham groups were significantly lower than those in the placebo group $(\mathrm{P}<0.05$, Figure 3), These results indicate that Cur also has a good protective effect on a variety of organs other than the kidney..

\section{Cur downregulates the inflammatory response induced by SAKI}

The medical community generally believes that a continuously activated immune response is the major cause of renal function damage in patients with SAKI (17). In the SD rat model of SAKI, We found significant increases in serum levels of pro-inflammatory cytokines TNF- $\alpha$ and IL10 in the placebo group $(\mathrm{P}<0.05)$, which is consistent with the phenomenon reported in clinical samples, while the levels of TNF- $\alpha$ and IL-10 in curcumin treatment group and sham operation group were significantly lower than those in placebo group $(\mathrm{P}<0.05$, Figure 4$)$. This indicated that the progression of SAKI may be related to the overcontinuous activation of an immune response in patients.

\section{Cur inhibits the expression profiles of related markers during SAKI progression}

According to previous studies, NGAL, serum CysC, and KIM-1 can be used as accurate and reliable markers for the early diagnosis of SAKI (18), so Cur may change the molecular expression level of these markers during SAKI progression. The levels of NGAL, CysC, and KIM-1 in the serum samples of the placebo group were significantly higher than those in the sham group $(\mathrm{P}<0.05)$. These parameters were significantly lower in the curcumin group compared with the placebo group $(\mathrm{P}<0.05$, Figure 5), validating that NGAL, CysC, and KIM-1 can be used to judge the progression of SAKI. The therapeutic effect of Cur on this disease can also be evaluated by these markers.

\section{Cur inbibits TLR9 upregulation induced by SAKI}

Studies have shown that the occurrence of septic acute kidney injury leads to significant up-regulation of TLR9 (19). Cur, as a vital inhibitor of TLR (20), may exert its therapeutic effect on SAKI by inhibiting the TLR9 signaling pathway. In this study, the level of TLR9 in the serum samples from the placebo group was significantly higher than that in the sham and curcumin groups $(\mathrm{P}<0.05$, Figure 6), which provides preliminary confirmation of our hypothesis.

\section{Cur exerts its therapeutic effect on SAKI by inbibiting the TLR9 signaling patbway}

The levels of UN, Cr, ALT, AST, amylase, CK, LDH, NGAL, KIM-1, CysC, and other disease-related factors in the curcumin group and TLR9-inhibitor group were significantly lower than those in the control inhibitor group $(\mathrm{P}<0.05$, Figure 7). In addition, the expression levels of the MyD88, IRF5, and IRF7 downstream molecules of the TLR9 signaling pathway in the curcumin group were found to be similar to those in the TLR9-inhibitor group and were significantly lower than those in the control-inhibitor group $(\mathrm{P}<0.05$, Figure 8$)$. The results indicated that Cur exerted its therapeutic effect on SAKI by inhibiting the TLR9 signaling pathway.

\section{Discussion}

Sepsis-induced AKI (SAKI) has been reported to be caused by major surgery, heart failure, respiratory failure, 

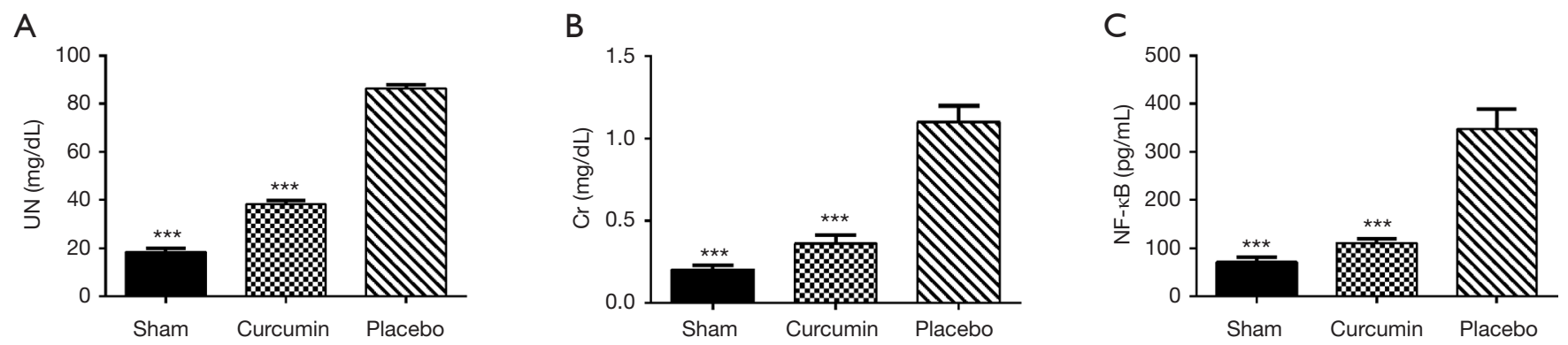

Figure 2 Cur significantly alleviates SAKI-induced renal dysfunction. The serum levels of UN, Cr, and NF- $\kappa B$ in SD rats were detected 24 hours later as indexes to evaluate renal function. (A) Urine nitrogen level in serum; (B) creatinine level in serum; (C) NF- $\kappa \mathrm{B}$ level in serum. *** indicates $\mathrm{P}<0.001$, with the placebo group as the control. SAKI, septic acute kidney injury; UN, urea nitrogen; Cr, creatinine; SD, Sprague-Dawley.
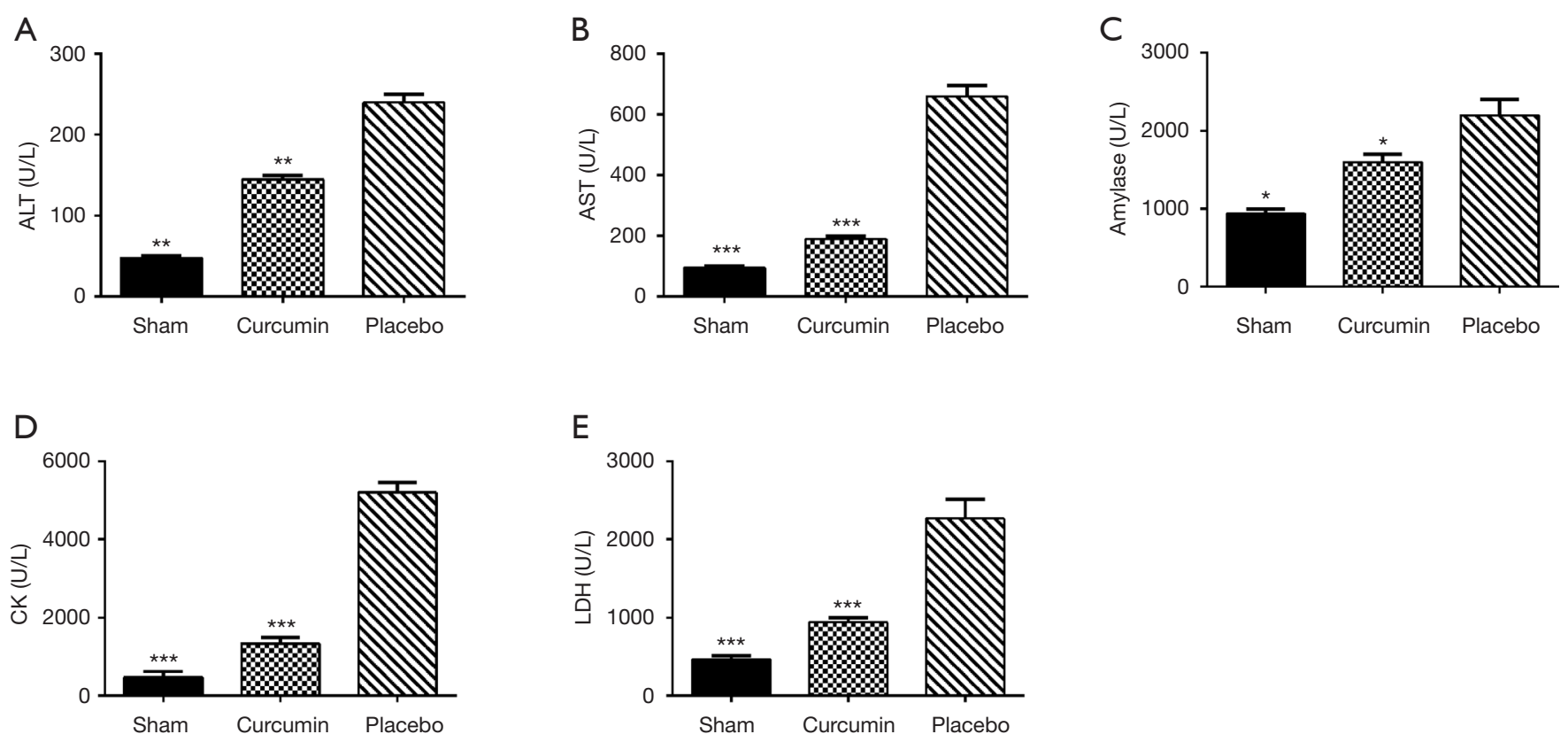

Figure 3 Cur significantly relieves SAKI-induced multiple organ damage. The serum levels of ALT, AST, amylase, CK, and LDH in SD rats were detected 24 hours later as indexes to evaluate the function of multiple organs. (A) ALT level; (B) serum AST level; (C) serum amylase level; (D) serum CK level; (E) serum LDH level. * indicates $\mathrm{P}<0.05$, ** indicates $\mathrm{P}<0.01$, ** indicates $\mathrm{P}<0.001$ with the placebo group as the control. Cur, curcumin; SAKI, septic acute kidney injury; ALT, alanine aminotransferase; AST, aspartate aminotransferase; CK, creatine kinase; LDH, lactate dehydrogenase; SD, Sprague-Dawley.

hypovolemia, and tissue hypoperfusion (21). According to recent studies, microcirculatory dysfunction, inflammation, and bio-energetic adaptive response to injury are also contribute to SAKI pathogenesis (22). Due to the insufficient understanding of the pathophysiology of SAKI, there is no guaranteed treatment for SAKI. Based on the mechanisms of SAKI we have got, fluid resuscitation, systematic resuscitation have been used clinically, but the most common therapeutic protocol was Renal replacement therapy (RRT), while the best timing to implement is hard to control (23), thus we should go deeper to explore the mechanism of SAKI. 

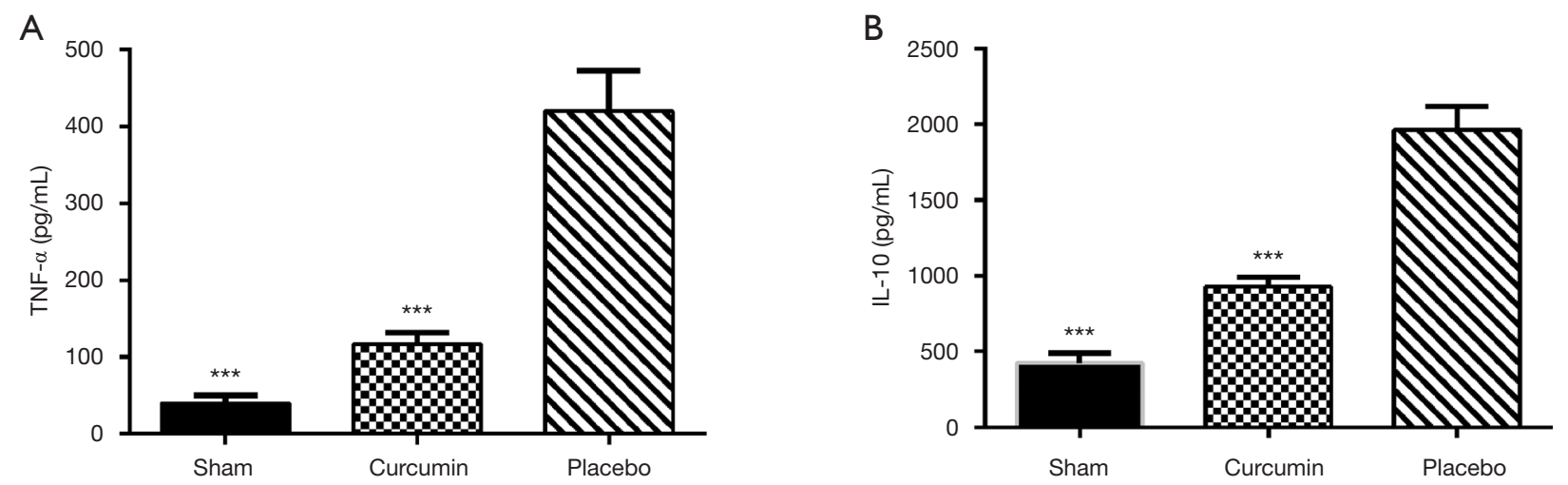

Figure 4 Cur significantly inhibits the upregulation of serum immune factors induced by SAKI. The levels of TNF- $\alpha$ and IL-10 in the serum samples of SD rats were measured 24 hours later. (A) TNF- $\alpha$ level in serum samples; (B) L-10 level in serum samples. *** indicates $\mathrm{P}<0.001$, with the placebo group as the control. SAKI, septic acute kidney injury; SD, Sprague-Dawley.
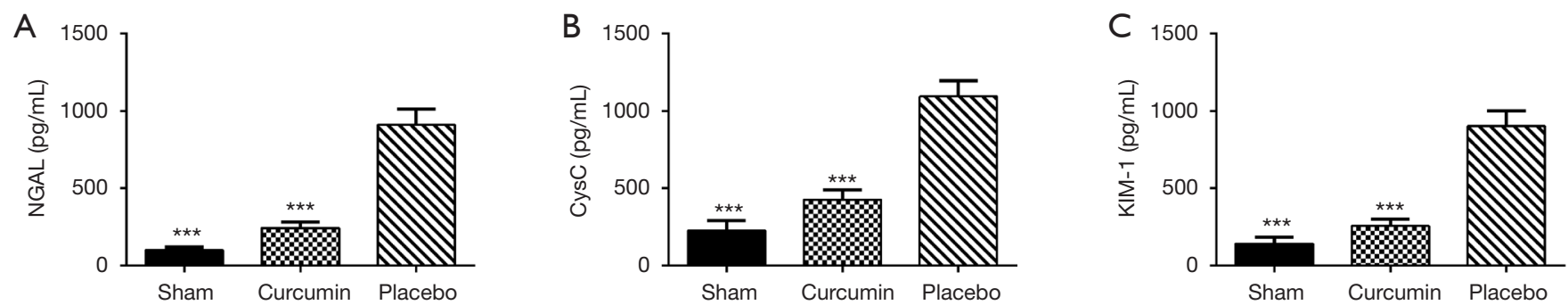

Figure 5 Cur significantly reduces the expression of NGAL, CysC, and KIM-1. The molecular expression levels of NGAL, CysC, and KIM-1 in the serum samples of SD rats were measured 24 hours later. (A) NGAL level in the serum samples of the different treatment groups; (B) CysC level in the serum samples of the different treatment groups; (C) KIM-1 level in the serum samples of the different treatment groups. *** indicates $\mathrm{P}<0.001$, with the placebo group as the control. NGAL, neutrophil gelatinase-associated lipocalin; CysC, cystatin-C; KIM-1, kidney injury molecule-1; SD, Sprague-Dawley.

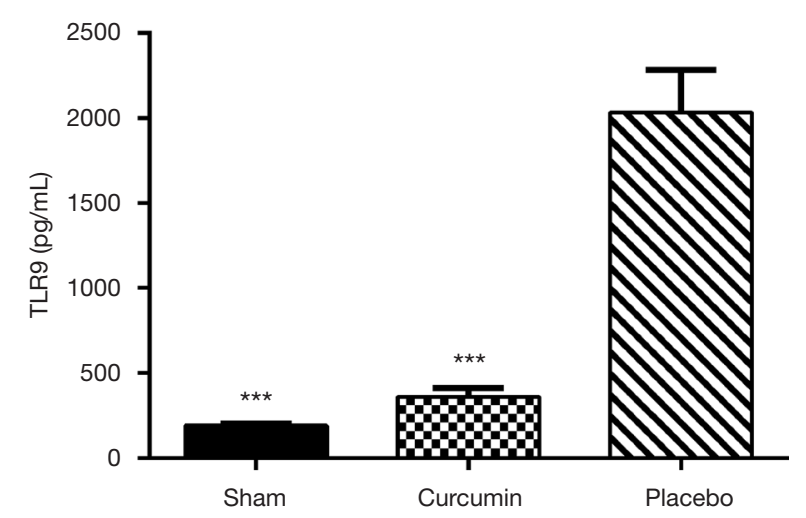

Figure 6 Cur downregulates TLR9 overexpression induced by SAKI. The TLR9 expression in the serum samples of SD rats was detected 24 hours later. ${ }^{* * *}$ indicates $\mathrm{P}<0.001$, with the placebo group as the control. Cur, curcumin; SAKI, septic acute kidney injury; SD, Sprague-Dawley.
According to the existing literature, Cur has antiinfective properties against various human pathogens, such as the influenza virus, hepatitis $\mathrm{C}$ virus, and HIV. It can also reduce kidney damage associated with rhabdomyolysis (24). Of note, the most promising application potential is its anti-inflammatory effect, and many clinical studies have evaluated its biological activity under various inflammatory conditions. These clinical trial data not only show the actual therapeutic effect of curcumin on some specific diseases, but also indicate that Cur has considerable safety and tolerability in human body, which provides very valuable experience for clinical trials of Cur in SAKI.

In clinical sample reports, SAKI often induces injury to multiple organs, such as the lungs, heart, brain, liver, and kidneys (25). By detecting the concentration changes of related factors in our rat model, we found that SAKI caused 

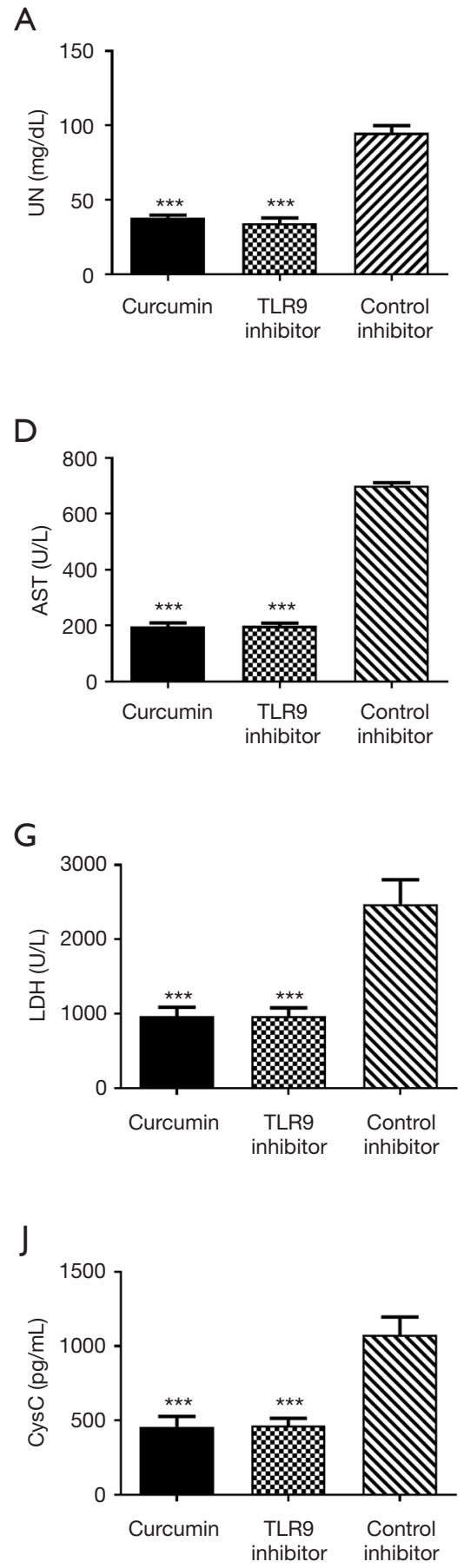

B

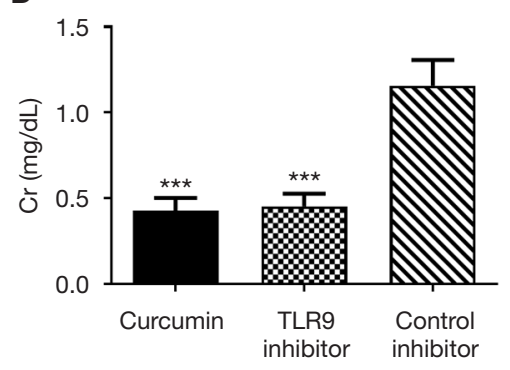

E

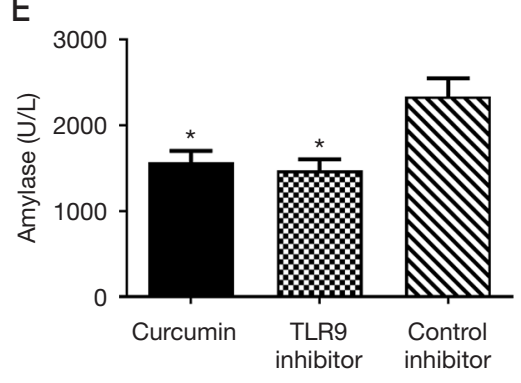

$\mathrm{H}$

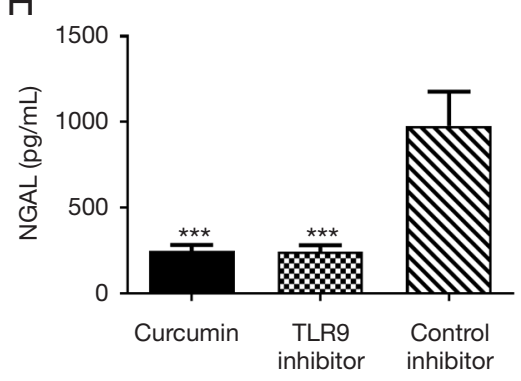

C

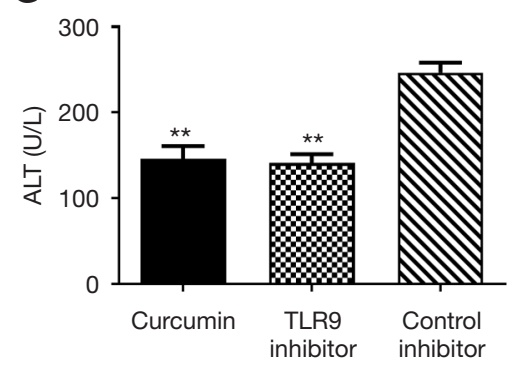

$\mathrm{F}$

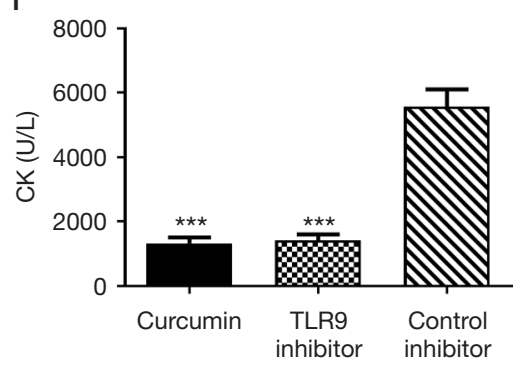

I

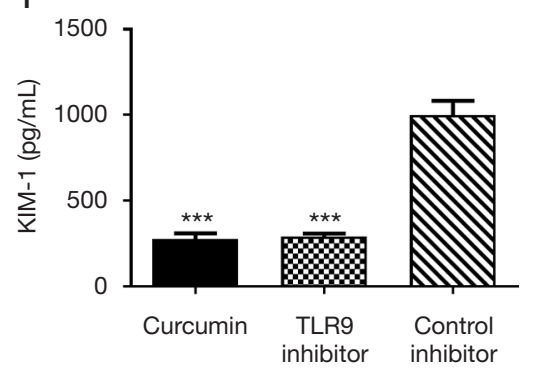

Figure 7 TLR9 inhibitor significantly downregulates the molecular expression level of SAKI-related factors. Six hours after CLP, SD rats were orally administered with $\mathrm{Cur}(\mathrm{n}=8,40 \mathrm{mg} / \mathrm{kg}$ ) and equal doses of the TLR9 inhibitor and control drug, respectively, and the expression levels of UN (A), Cr (B), ALT (C), AST (D), amylase (E), CK (F), LDH (G), NGAL (H), KIM-1 (I), and CysC (J) in the serum samples were detected 24 hours later. * indicates $\mathrm{P}<0.05$, ** indicates $\mathrm{P}<0.01$, ${ }^{* * *}$ indicates $\mathrm{P}<0.001$, with the control inhibitor group as the control. CLP, cecal ligation perforation; SD, Sprague-Dawley; SAKI, septic acute kidney injury; Cur, curcumin; UN, urea nitrogen; Cr, creatinine; ALT, alanine aminotransferase; AST, aspartate aminotransferase; CK, creatine kinase; LDH, lactate dehydrogenase; NGAL, neutrophil gelatinase-associated lipocalin; KIM-1, kidney injury molecule-1; CysC, cystatin-C. 

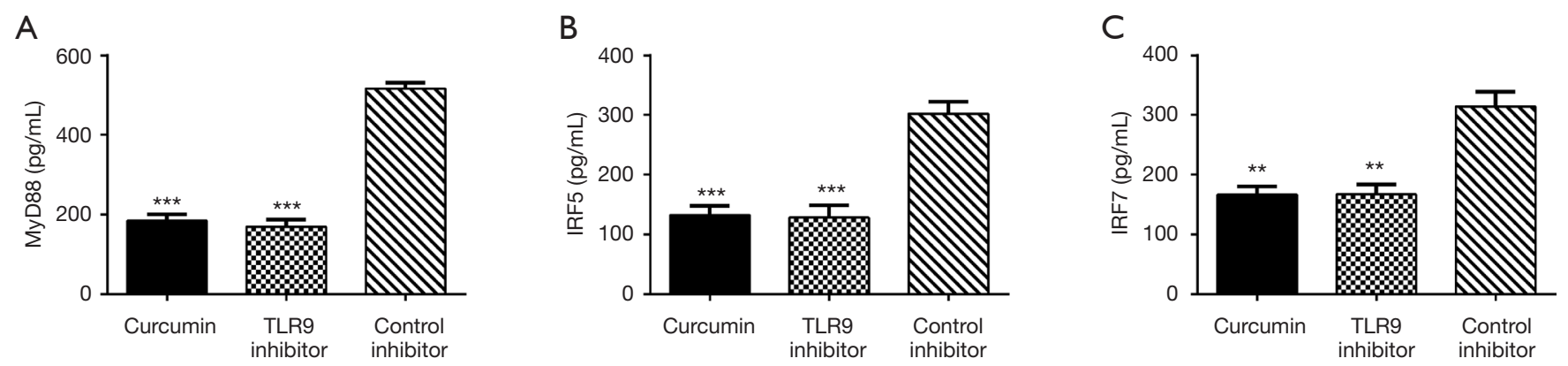

Figure 8 Cur inhibits activation of the TLR9 signaling pathway induced by SAKI. SD rats were orally administered with Cur ( $\mathrm{n}=8,40 \mathrm{mg} / \mathrm{kg}$ ) and equal doses of the TLR9 inhibitor and control drug 6 hours after CLP, and the expression levels of MyD88, IRF5, and IRF7 (important factors of the TLR9 signaling pathway) were detected in the serum samples 24 hours later (A) MyD88 level in the serum samples of the different treatment groups; (B) IRF5 level in the serum samples of the different treatment groups; (C) IRF7 level in the serum samples of the different treatment groups. ${ }^{* *}$ indicates $\mathrm{P}<0.01$, ${ }^{* *}$ indicates $\mathrm{P}<0.001$, with the sham group as the control. SAKI, septic acute kidney injury; $\mathrm{SD}$, Sprague-Dawley; Cur, curcumin; CLP, cecal ligation perforation.

damage to the kidneys and other multiple organs. This indicates that the animal model simulates the development of SAKI in human body to a certain extent, and provides a reliable theoretical basis for future research and clinical drug experiments. Besides, we found that Cur significantly reduced the incidence of multiple organ damage. Therefore, we speculate that Cur may play an important role in balancing the immune response of the body.

Previous studies have shown that Cur can significantly downregulate the expression level of inflammatory factors such as TNF- $\alpha$, IL-1, IL-2, IL-6, IL-8, and mitogenactivated protein kinase (MAPK) (26). Cur can also inhibit the activation of the extracellular TLR2/4 signaling pathway and can suppress the activation of IRAK1, IRAK6, TRIF, and MyD88 downstream of the TLR2/4 signaling pathway. In addition, Cur has been proved to be one of the antagonists of the intracellular TLR9 signaling pathway (27). The change in TLR9 expression is usually considered to be a sign of the development of various inflammation-related diseases, while Cur can reduce TLR9 expression in the rat liver and act as an antiinflammatory and immunomodulatory agent to treat liver inflammation (28). The most recent study has revealed that Cur could ameliorate the SAKI induced by CLP, but they only detected the kidney protective effect of the Cur (29). While in this paper, we have found that the Cur could significantly decrease the mortality caused by AKI, and we also determined that Cur could prevent multiple organs from dysfunction caused by AKI, meanwhile, our results were almost based on the in vivo data, which may be more coincidence with the pathogenesis of AKI in human.

\section{Conclusions}

In this study, we found that the expression of proinflammatory factors, which rose significantly in rats with SAKI, can be counteracted by Cur. Also, the activation of the TLR9 molecule was detected in the SAKI rat model, but not in the curcumin group or the control-inhibitor group. The relevant results of this study showed that Cur, while playing its role in the resistance to SAKI, indeed inhibited the expression of downstream molecules in the TLR9 signaling pathway. Based on the above results, Cur can inhibit SAKI by inhibiting the activation of the TLR9 signaling pathway.

\section{Acknowledgments}

Funding: This study was funded by the Clinical Research Fund Project of Qiqihar Academy of Medical Sciences (QMSI2019L-10).

\section{Footnote}

Reporting Checklist: The authors have completed the ARRIVE reporting checklist. Available at http://dx.doi. org/10.21037/tau-21-385

Data Sharing Statement: Available at http://dx.doi. org/10.21037/tau-21-385 
Conflicts of Interest: All authors have completed the ICMJE uniform disclosure form (available at http://dx.doi. org/10.21037/tau-21-385). The authors have no conflicts of interest to declare.

Etbical Statement: The authors are accountable for all aspects of the work in ensuring that questions related to the accuracy or integrity of any part of the work are appropriately investigated and resolved. Experiments were performed in compliance with Qiqihar Medical University guidelines for the care and use of animals. The study was approved by the Qiqihar Medical University Institutional Review Board (No. QMU-AECC-2018-32), Heilongjiang, China.

Open Access Statement: This is an Open Access article distributed in accordance with the Creative Commons Attribution-NonCommercial-NoDerivs 4.0 International License (CC BY-NC-ND 4.0), which permits the noncommercial replication and distribution of the article with the strict proviso that no changes or edits are made and the original work is properly cited (including links to both the formal publication through the relevant DOI and the license). See: https://creativecommons.org/licenses/by-nc-nd/4.0/.

\section{References}

1. Sun J, Zhang J, Tian J, et al. Mitochondria in SepsisInduced AKI. J Am Soc Nephrol 2019;30:1151-61.

2. Diakowska D, Nienartowicz M, Grabowski K, et al. Tolllike receptors TLR-2, TLR-4, TLR-7, and TLR-9 in tumor tissue and serum of the patients with esophageal squamous cell carcinoma and gastro-esophageal junction cancer. Adv Clin Exp Med 2019;28:515-22.

3. Balka KR, De Nardo D. Understanding early TLR signaling through the Myddosome. J Leukoc Biol 2019;105:339-51.

4. Xia S, Lin H, Liu H, et al. Honokiol Attenuates SepsisAssociated Acute Kidney Injury via the Inhibition of Oxidative Stress and Inflammation. Inflammation 2019;42:826-34.

5. Fanelli C, Arias SCA, Machado FG, et al. Innate And Adaptive Immunity are Progressively Activated in Parallel with Renal Injury in the 5/6 Renal Ablation Model. Sci Rep 2017;7:3192.

6. Mirzaei H, Shakeri A, Rashidi B, et al. Phytosomal curcumin: A review of pharmacokinetic, experimental and clinical studies. Biomed Pharmacother 2017;85:102-12.
7. Asadi S, Gholami MS, Siassi F, et al. Beneficial effects of nano-curcumin supplement on depression and anxiety in diabetic patients with peripheral neuropathy: A randomized, double-blind, placebo-controlled clinical trial. Phytother Res 2020;34:896-903.

8. Qin S, Huang L, Gong J, et al. Efficacy and safety of turmeric and curcumin in lowering blood lipid levels in patients with cardiovascular risk factors: a meta-analysis of randomized controlled trials. Nutr J 2017;16:68.

9. Azhdari M, Karandish M, Mansoori A. Metabolic benefits of curcumin supplementation in patients with metabolic syndrome: A systematic review and metaanalysis of randomized controlled trials. Phytother Res 2019;33:1289-301.

10. Salehi B, Stojanović-Radić Z, Matejić J, et al. The therapeutic potential of curcumin: A review of clinical trials. Eur J Med Chem 2019;163:527-45.

11. Lee DE, Lee SJ, Kim SJ, et al. Curcumin Ameliorates Nonalcoholic Fatty Liver Disease through Inhibition of O-GlcNAcylation. Nutrients 2019;11:2702.

12. Wojcik M, Krawczyk M, Wojcik P, et al. Molecular Mechanisms Underlying Curcumin-Mediated Therapeutic Effects in Type 2 Diabetes and Cancer. Oxid Med Cell Longev 2018;2018:9698258.

13. Cunha Neto F, Marton LT, de Marqui SV, et al. Curcuminoids from Curcuma Longa: New adjuvants for the treatment of crohn's disease and ulcerative colitis? Crit Rev Food Sci Nutr 2019;59:2136-43.

14. Momtazi-Borojeni AA, Haftcheshmeh SM, Esmaeili SA, et al. Curcumin: A natural modulator of immune cells in systemic lupus erythematosus. Autoimmun Rev 2018;17:125-135.

15. Post EH, Kellum JA, Bellomo R, et al. Renal perfusion in sepsis: from macro-to microcirculation. Kidney Int 2017;91:45-60.

16. Yasuda H, Leelahavanichkul A, Tsunoda S, et al. Chloroquine and inhibition of Toll-like receptor 9 protect from sepsis-induced acute kidney injury. Am J Physiol Renal Physiol 2008;294:F1050-F1058.

17. Jang HR, Rabb H. Immune cells in experimental acute kidney injury. Nat Rev Nephrol 2015;11:88.

18. Assadi F, Sharbaf FG. Urine KIM-1 as a potential biomarker of acute renal injury after circulatory collapse in children. Pediatr Emerg Care 2019;35:104-7.

19. Liu $\mathrm{L}, \mathrm{Li} Y, \mathrm{Hu} Z$, et al. Small interfering RNA targeting Toll-like receptor 9 protects mice against polymicrobial septic acute kidney injury. Nephron Exp Nephrol 2012;122:51-61. 
20. Boozari M, Butler AE, Sahebkar A. Impact of curcumin on toll-like receptors. J Cell Physiol 2019;234:12471-82.

21. Zarbock A, Gomez H, Kellum JA. Sepsis-induced acute kidney injury revisited: pathophysiology, prevention and future therapies. Curr Opin Crit Care 2014;20:588-95.

22. Zhang Z. Biomarkers, diagnosis and management of sepsis-induced acute kidney injury: a narrative review. Heart Lung Vessel 2015;7:64-73.

23. Bagshaw SM, Wald R, Adhikari NKJ, et al. Timing of Initiation of Renal-Replacement Therapy in Acute Kidney Injury. N Engl J Med 2020;383:240-51.

24. Nelson KM, Dahlin JL, Bisson J, et al. The Essential Medicinal Chemistry of Curcumin. J Med Chem 2017;60:1620-37.

25. Husain-Syed F, Rosner MH, Ronco C. Distant organ dysfunction in acute kidney injury. Acta Physiol (Oxf) 2020;228:e13357.

26. Varma SR, Sivaprakasam TO, Mishra A, et al. Imiquimod-

Cite this article as: Li H, Sun H, Xu Y, Xing G, Wang X. Curcumin plays a protective role against septic acute kidney injury by regulating the TLR9 signaling pathway. Transl Androl Urol 2021;10(5):2103-2112. doi: 10.21037/tau-21-385 induced psoriasis-like inflammation in differentiated Human keratinocytes: Its evaluation using curcumin. Eur J Pharmacol 2017;813:33-41.

27. Dai J, Gu L, Su Y, et al. Inhibition of curcumin on influenza A virus infection and influenzal pneumonia via oxidative stress, TLR2/4, p38/JNK MAPK and NF-кB pathways. Int Immunopharmacol 2018;54:177-87.

28. She L, Xu D, Wang Z, et al. Curcumin inhibits hepatic stellate cell activation via suppression of succinateassociated HIF-1 $\alpha$ induction. Mol Cell Endocrinol 2018;476:129-38.

29. Zhu H, Wang X, Wang X, et al. Curcumin attenuates inflammation and cell apoptosis through regulating $\mathrm{NF}-\kappa \mathrm{B}$ and JAK2/STAT3 signaling pathway against acute kidney injury. Cell Cycle 2020;19:1941-51.

(English Language Editor: D. Fitzgerald) 